\title{
Jules Duchastel
}

sociologue, département de sociologie, UQAM

1979

\section{"La contre-culture, une idéologie de l'apolitisme"}

Un document produit en version numérique par Jean-Marie Tremblay, bénévole, professeur de sociologie au Cégep de Chicoutimi

Courriel: imt sociologue@,videotron.ca

Site web pédagogique : $\underline{\text { http://www.uqac.ca/jmt-sociologue }}$

Dans le cadre de la collection: "Les classiques des sciences sociales"

Site web: http://www.uqac.ca/Classiques des_sciences_sociales

Une collection développée en collaboration avec la Bibliothèque Paul-Émile-Boulet de l'Université du Québec à Chicoutimi

Site web: http://bibliotheque.uqac.uquebec.ca/index.htm 
Cette édition électronique a été réalisée par Jean-Marie Tremblay, bénévole, professeur de sociologie au Cégep de Chicoutimi à partir de :

Jules Duchastel, "La contre-culture, une idéologie de l'apolitisme". Un article publié dans l'ouvrage intitulé La transformation du pouvoir au Québec. Actes du colloques de l'ACSALF, 1979, pp. 253-264. Montréal: Éditions Albert SaintMartin, 1980, 378 pp.

[Autorisation accordée par l'auteur, M. Jules Duchastel, sociologue à l'UQAM, le 5 janvier 2004.]

20 Courriel : duchastel.jules@uqam.ca

Polices de caractères utilisés :

Pour le texte: Times, 12 points.

Pour les citations : Times 10 points.

Pour les notes de bas de page : Times, 10 points.

Édition électronique réalisée avec le traitement de textes Microsoft Word 2001 pour Macintosh.

Mise en page sur papier format

LETTRE (US letter), 8.5' x 11 '’)

Édition réalisée le 26 juin 2004 à Chicoutimi, Québec.

Édition numérique complétée le 19 décembre 2004 à Chicoutimi, Ville de Saguenay, province de Québec, Canada. 


\section{Jules Duchastel}

sociologue, UQAM

\section{"La contre-culture, une idéologie de l'apolitisme"}

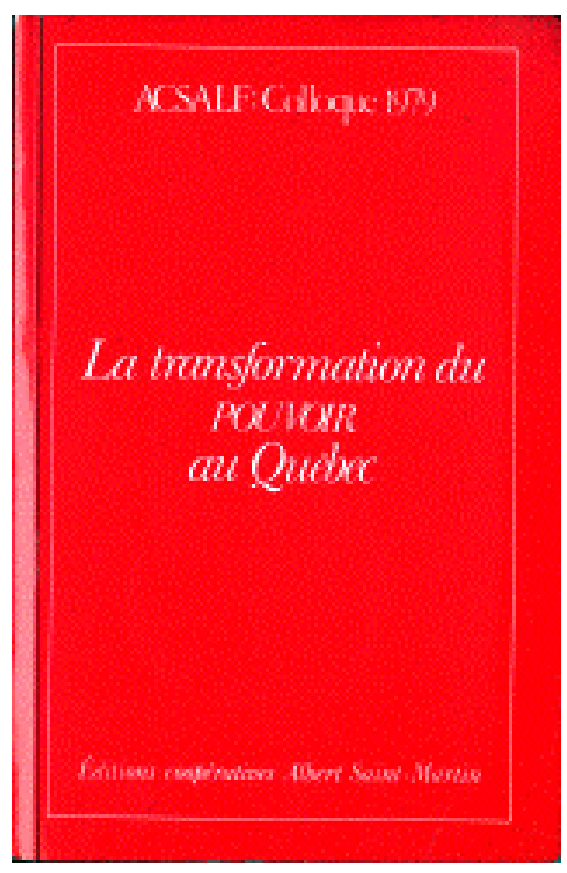

Un article publié dans La transformation du pouvoir au Québec. Actes du colloques de l'ACSALF, 1979, pp. 253-264. Montréal: Éditions Albert Saint-Martin, 1980, 378 pp. [Autorisation accordée par l'auteur le 5 janvier 2004.] 


\section{Table des matières}

1. Remarques préliminaires

a) Les notions de mouvement, de contre-culture et de pouvoir

b) Comment aborder le phénomène de la contre-culture

c) Apolitisme et idéologie de l'apolitisme

2. La contre-culture est une idéologie

a) Formation idéologique d'ensemble

b) Aspects critiques et intégristes

\section{La contre-culture est un mouvement social}

\section{Conclusion}




\section{Remarques préliminaires}

La contre-culture a représenté trop de significations à divers niveaux du vécu pour un grand nombre de personnes pour qu'il soit facile d'en parler sans entrainer une certaine confusion des termes. Il me semble donc nécessaire de commencer par certaines remarques visant à clarifier les diverses notions qui sont utilisées couramment pour aborder cette question et à expliquer la perspective dans laquelle je me situe.

\section{a) Les notions de mouvement, de contre-culture et de pouvoir}

\section{$\underline{\text { Retour à la table des matières }}$}

Le titre de l'atelier : «Le mouvement de la contre-culture face au pouvoir» introduit trois notions qu'il faut interroger pour éviter d'être prisonniers de représentations, sinon spontanées, du moins semi-construites et de reproduire d'une certaine façon - à un second degré - un discours autojustificateur. D'abord, quel est le sens de la notion de «mouvement » lorsqu'on l'associe à la contre-culture. On doit noter que dans les années 60 , le terme «mouvement » s'est souvent substitué à celui de contre-culture pour désigner cette expérience qui se voulait une alternative globale à la société. Cette notion inscrite à l'intérieur du discours contre-culture] visait à identifier l'aspect dynamique de la contre-culture, sa dimension de projet, mais aussi le caractère supposé de son irrémédiabilité. Le «mouvement» était donc un opérateur idéologique, principe de ralliement, facteur de mobilisation. Lorsqu'on parle ici de mouvement, ne s'en réfère-t-on pas plutôt à un concept sociologique construit, celui de mouvement social ? Dans ce 
cas, il apparaît nécessaire de changer de terrain. Il faudra plutôt se demander quels sont le moment, les conditions et les formes de son émergence et de son épanouissement.

De la même manière la notion de contre-culture pose un problème. Le texte de présentation de l'atelier parle de pratiques et de valeurs nouvelles. Ceci est vrai à un premier niveau descriptif. Mais, comme je le disais plus haut, c'est en plus un projet élaboré à travers ces pratiques visant une transformation, du moins dans la mesure de la définition que se donne la contre-culture d'elle-même. Elle se définit comme projet. Paradoxalement - vu le titre de mon exposé - je dirais qu'elle se donne comme projet politique. Cependant, si l'on change de terrain, on peut s'interroger sur la signification de ce néologisme, la contre-culture. À un premier niveau, il renvoie à la notion de culture, ce qui n'a pas pour effet de diminuer son ambiguïté. En effet, la notion de culture me semble être démunie de sens à force d'être investie d'une multitude de significations. À l'origine, la culture désignait le travail - à travers des formes spécifiques et un procès déterminé - mis en oeuvre pour extirper la subsistance de la terre. De façon imagée, la culture a aussi désigné le travail Produit au niveau de «l'esprit ». Il s'agit alors de la culture «cultivée ». Puis, une certaine « démocratisation» de la notion se produit avec l'extension du sens de la culture dans l'acception anthropologique. La culture y désigne un ensemble de manières d'être, de penser et d'agir. Enfin, avec la constitution d'une théorie sociologique - le structuro-fonctionnalisme - la notion de culture ne sert plus seulement à décrire les caractéristiques diverses des sociétés, mais devient principe d'explication en dernière analyse. La culture comme consensus devient l'explication de la pérennité relative des structures sociales. Dans cette acception même, la culture prend des contours imprécis. On ne sait trop ce qu'elle est, mais elle permet d'expliquer sur un mode globaliste et non conflictuel l'ensemble de la réalité sociale.

De quoi s'agit-il alors lorsque l'on parle de contre-culture ? Si la culture est consensus, peut-on parler de la contre-culture comme rupture de consensus ? D'une certaine façon, elle se propose comme alternative au consensus. Mais cette notion ne quitte pas le terrain d'une explication consensuelle-globalisante et non conflictuelle. À la limite, la notion de contre-culture s'appuie sur le relativisme des analyses culturalistes. Elle permet par ailleurs de laisser transparaître la nécessité de l'analyse dynamique. Mais elle retombe dans les mêmes travers que l'analyse culturaliste en ce qu'elle recherche un principe explicatif globaliste à travers une nouvelle forme consensuelle. Qu'il suffise d'indiquer dans ces remarques préliminaires qu'autant la notion de culture que celle de contre-culture présentent d'un côté une grande part d'ambiguïté et d'un autre côté s'appuient sur un postulat de consensualité. En contrepartie, il me semble pertinent de proposer le concept de pratique idéologique - discursive ou non discursive - pour rendre compte du mouvement de la contre-culture des années 60 . J'y reviendrai.

Le troisième terme du titre de l'atelier concerne le pouvoir. La définition de l'atelier distingue deux niveaux de pouvoir, soit le niveau interindividuel et le niveau sociétal. Je trouve acceptable cette distinction pour définir le niveau de l'approche. Cependant, encore une fois, il est possible de distinguer la définition spontanée du pouvoir d'une définition construite théoriquement. Que l'on pose le problème du pouvoir comme principe 
d'opposition politique ou comme « objet idéologique » à travailler - on peut, par exemple, repenser les rapports de pouvoir dans une commune - il me semble nécessaire de définir ce qu'on entend par pouvoir sur le plan théorique. Une opposition - nécessairement réductrice - indiquera l'insuffisance de cette définition. D'un côté, on définit le premier comme un universel. Il s'agit d'une essence plus ou moins philosophique ou psychologique qui traverse l'ensemble du «corps social»-du microcosme au macrocosme. Cette représentation d'un enjeu universel détermine souvent une position anarchiste sur le plan politique et un point de vue microsocial par rapport au champ d'intervention. D'un autre côté, on lie le pouvoir à l'exercice d'une exploitation et d'une domination spécifique dans des rapports sociaux historiquement déterminés. Cette représentation pose le problème du pouvoir à travers une théorie de l'État comme lieu de rapports de force et de luttes. Cette représentation entraîne une position politique axée sur l'analyse des contradictions et des luttes et une perspective plus macroscopique au niveau de l'intervention.

Le sens de ces premières remarques vise à établir que bien que le discours idéologique et le discours théorique ne soient pas totalement indépendants - et, au contraire, ils sont inextricablement articulés - il faut savoir établir une distance minimale entre les pratiques et discours analysés et le discours de l'analyse.

\section{b) Comment aborder le phénomène de la contre-culture}

\section{$\underline{\text { Retour à la table des matières }}$}

La seconde remarque concerne le niveau de l'approche que je suggérerai plus loin du phénomène de la contre-culture. Ce niveau est dépendant des définitions théoriques que je donne, des pratiques idéologiques, des mouvements sociaux et de l'État. Dans tous les cas, ces définitions se rapportent à l'ensemble des rapports sociaux. Pour moi, les pratiques idéologiques doivent être comprises dans le cadre du fonctionnement idéologique général d'une formation sociale, laquelle contribue à la reproduction / transformation des rapports sociaux (cette dernière devant être comprise dans le cadre d'un rapport de force entre classes sociales). Les mouvements sociaux représentent la forme active que prennent ces luttes aux divers points de friction des formations sociales. Enfin, l'État est la condensation de l'ensemble de ces rapports de force et de ces luttes. De ce point de vue, l'approche du phénomène de la contre-culture est fixée à un niveau général d'explication. Cela ne veut pas dire que ces concepts excluent un niveau d'analyse plus microscopique. Il faudrait cependant qu'ils soient remodelés en fonction d'une approche du micro-social et que les articulations entre ce niveau et le niveau de l'analyse plus général soient élaborées. Il ne sera donc pas question ici d'expériences particulières 
(écoles libres, communes, nouvelle agriculture, production artisanale, etc.). De plus j'exclus de mon analyse, ici, le cas de mouvements politiques plus spécifiques - et relativement marginaux - qui ont tenté de faire le pont entre l'idéologie contre-culturelle et le politique (mouvements libertaires ou anarchistes). Je préfère aborder la question dans la perspective du développement historique du capitalisme dans sa présente phase monopoliste. Celui-ci se manifeste par une profonde transformation de l'État, une modification de la structure des classes et une réorganisation de la configuration idéologique d'ensemble. Je m'appuierai pour développer mes thèses sur les mouvements sociaux des années 60 et sur le discours idéologique explicite de ceux qui se sont réclamés de la contre-culture.

\section{c) Apolitisme et idéologie de l'apolitisme}

\section{$\underline{\text { Retour à la table des matières }}$}

Le titre de ma communication, « la contre-culture, une idéologie de l'apolitisme », demande quelques explications. Il me semble nécessaire de souligner qu'il n'y a pas à proprement parler d'idéologie apolitique. Ce n'est pas la même chose que de parler d'une idéologie de l'apolitisme. En effet, de larges secteurs du mouvement contreculturel des années 60 se sont explicitement

Pouvoir et culture 257

réclamés de l'apolitisme. Il n'en demeure pas moins que ce mouvement fut politique dans ses effets. J'ajoute que les mouvements plus contemporains qui puisent largement leur inspiration dans l'idéologie contre-culturelle des années soixante, tout en voulant réintroduire le politique dans leur plate-forme, continuent à produire l'effet politique à travers le mécanisme de l'apolitisme. Cela implique que je définisse l'apolitisme. Celuici est une pratique idéologique dont l'effet politique est produit à travers une décentration de l'action politique hors du lieu où les rapports de force se nouent et se dénouent ordinairement. L'apolitisme nie le caractère politique de son intervention en prétendant désinvestir le lieu traditionnel de son exercice et en visant le rapport d'articulation entre les contradictions secondaires où il se concentre et les contradictions principales autour du pouvoir de l'État. 


\section{La contre-culture est une idéologie}

$\underline{\text { Retour à la table des matières }}$

Il faut préciser dès le départ que la contre-culture ne saurait être considérée comme mouvement social unifié, pas plus que comme idéologie unifiée. L'autodéfinition qu'elle donne d'elle-même tend en effet à la représenter sous une forme unifiée. Je crois plutôt qu'il faut considérer toutes les manifestations de ce mouvement dans leur multiplicité même et sous l'angle de leur émergence historique. Il n'en demeure pas moins que certaines convergences peuvent être observées au niveau d'un discours idéologique en marge de ces manifestations. Je propose de nommer cette convergence tendance idéologique contre-culturelle.

Une seconde précision s'avère utile. Il est souvent dit que la contre-culture a été récupérée socialement. Si cette notion de récupération permet, d'une certaine manière, d'indiquer la puissance d'un système qui sait assimiler les moindres parcelles de critique, elle est intéressante. Par contre, cette notion a le désavantage de laisser croire en la virginité supposée de ce qui est récupéré. En effet, dans cette perspective, la contreculture est considérée en dehors des conditions sociales de son émergence dans un premier temps et comme détournée par ces mêmes conditions dans un second temps. Il me semble plus utile d'en analyser l'émergence au cœur même des rapports sociaux, ce qui n'empêche pas d'en saisir les aspects critiques.

Ceci m'amène à indiquer que le discours contre-culturel n'émerge pas isolé dans l'ensemble de la configuration idéologique d'une formation sociale. Il est intéressant de noter que certaines catégories d'analyse du social se développent à divers niveaux de la structure sociale. Dans le domaine des sciences sociales, le développement d'une sousdiscipline de la sociologie (la sociologie de la jeunesse) et d'une certaine perspective théorique pour l'explication des sociétés (théorie de la société post-industrielle) s'articule aussi très bien avec les catégories discursives centrales de l'idéologie contre-culturelle ${ }^{1}$.

Voir Jules Duchastel, Théorie ou idéologie de la jeunesse : discours et mouvement social, Université de Montréal, 1978, thèse de doctorat. Voir également Idem, « The Sociology of Youth as the Theoretical Basis for Ideologies of the Counter-Culture », Uppsala, Association internationale de sociologie, 1978. 


\section{a) Formation idéologique d'ensemble}

$\underline{\text { Retour à la table des matières }}$

Dire que la contre-culture est une idéologie nécessite que l'on précise ce que l'on entend par idéologie. Cela impose un certain détour par une théorie plus générale des formations idéologiques. La fameuse distinction établie par Louis Althusser, entre l'idéologie en général et les idéologies concrètes ${ }^{2}$, demeure pertinente pour indiquer la nécessité de poser le problème du fonctionnement de l'idéologie dans nos formations sociales. Le problème des idéologies concrètes est nécessairement articulé à ce niveau fondamental, mais les contraintes de l'exposition autorisent à introduire cette question dans un deuxième temps.

Le fonctionnement idéologique dans les formations sociales capitalistes avancées peut être pensé à deux niveaux. De façon générale, il assure la reproduction / transformation des rapports sociaux. Il est aussi matérialisation - à travers des formes spécifiques - d'un rapport de force qui se manifeste dans des luttes pour la défense des intérêts de classe. Deuxièmement, au niveau de la pratique des agents, le fonctionnement idéologique constitue les individus en agents sociaux dont la forme d'assujettissement est marquée par leur place dans la lutte des classes. En ce sens, il n'existe pas d'individus qui ne soient marqués par un rapport social et donc constitués en agents sociaux. Dans le mode de production capitaliste, cette constitution est produite à travers l'interpellation des individus en sujets ${ }^{3}$. Le travail d'interpellation est largement produit à travers l'appareil de la famille, qui véhicule l'idéologie de la distinction entre vie privée et vie publique. La vie privée devient le lieu de développement de l'individualité. La vie publique permet de représenter les rapports sociaux sous une forme idéologique. Ce bref rappel sur le fonctionnement idéologique permet d'indiquer comment s'exécutent les fonctions et le procès de reproduction / transformation dans nos formations sociales. Il faut cependant recourir à des notions complémentaires pour analyser de plus près les rapports sociaux. J'examinerai dans ce sens quelle est la tendance idéologique qui domine dans la présente phase du développement capitaliste et quelle est la place d'une autre tendance idéologique qui s'articule à la première.

Louis Althusser, «Idéologie et appareils idéologiques d'État »m in La Pensée, no 51, Paris, juin 1970.

Il ne s'agit pas, selon moi, d'une interpellation universelle comme c'est le cas pour Althusser, mais de la forme spécifique que revêt la constitution des agents sociaux dans le mode de production capitaliste. 
Le développement de l'État dans la phase proprement monopoliste du capitalisme implique une croissance de son interventionnisme et, par voie de conséquence, une multiplication des points de friction (multiplication des contradictions secondaires, multiplication des lieux de lutte sociale). Le développement de l'État s'accompagne aussi d'une restructuration des rapports de classe. Le développement de ce qu'il est convenu d'appeler la nouvelle petite bourgeoisie en est une des conséquences principales ${ }^{4}$. Enfin, le progrès scientifique marque la troisième caractéristique de cette transformation. La tendance idéologique dominante que je qualifie de technocratique se construit sur ces trois tendances. L'idéologie de la distinction entre vie privée et vie publique, bien qu'encore opérationnelle, se voit de plus en plus contredite par les faits. La tendance technocratique m'apparaît susceptible de fournir la justification de l'envahissement du privé par le public. Elle le fait par le mode d'un triptyque idéologique : elle se fonde d'abord sur le progrès scientifique et technologique pour proposer la nécessité de la modernisation, elle prend assise sur la complexité croissante des mécanismes de régulation et de gestion du social pour imposer la rationalité comme unique mode de fonctionnement, enfin elle déduit de ce qui précède que le besoin de planification implique des pouvoirs accrus pour les « experts ». Il y a donc déplacement de la représentation « libérale » du bien commun vers une représentation de l'État interventionniste. Cette tendance idéologique permet de constater à un certain niveau l'importance de certaines pratiques de la nouvelle petite bourgeoisie dans la régulation des rapports sociaux.

La contre-culture peut être considérée comme étant une contre-tendance à cette tendance dominante dans les formations capitalistes avancées. Le premier élément pertinent me semble être la base de classe de cette idéologie. Les mouvements sociaux des années 60 ont surtout recruté leurs membres parmi les couches de la nouvelle petite bourgeoisie. (Ces mouvements ont souvent été articulés aux milieux étudiants, soit directement au niveau de la contestation dans les universités, soit indirectement par la clientèle des étudiants ou des drop-out.) Les produits contre-culturels (revues, création, artisanat) et les pratiques contre-culturelles (modes vestimentaires, habitudes de consommation, drogue, communes, manifestations) ont fait appel aux mêmes couches sociales. Il est donc intéressant de noter que les éléments de l'idéologie contre-culturelle s'élaborent en s'appuyant sur la même base sociale. Le deuxième élément d'intérêt est le fait que, comme nous le verrons, la critique du discours contre-culturel ou des mouvements contre-culturels identifie leur principe d'opposition sous la figure du discours et du pouvoir technocratiques. À un premier niveau d'analyse, il apparait clairement que les credos de cette tendance technocratique sont remis en question. Cependant, un troisième élément vient atténuer la portée des deux premiers. Si, du point de vue des positions idéologiques élaborées et des oppositions sur le terrain, la tendance contre-culturelle se présente comme contre-tendance de l'idéologie technocratique, il faut observer en même

La notion de nouvelle petite bourgeoisie est trop large pour être satisfaisante. Elle est définie négativement dans la mesure où elle regroupe les couches sociales qui ne se placent pas dans les rapports de production du point de vue des critères de la propriété ou du travail productif. Elle est donc multiple. Les fractions qui nous intéressent ici sont plutôt celles qui sont définies par leur fonction idéologico-politique dans les rapports sociaux et risquent par le fait même d'être davantage polarisées vers la bourgeoisie. 
temps que cette idéologie contre-culturelle continue à renforcer l'idéologie du sujet. De là, il me semble possible de proposer qu'en dernière analyse cette tendance, bien que présentant des aspects critiques vis-à-vis des rapports sociaux, favorise plus généralement leur reproduction. Un examen des aspects critiques et intégristes de cette idéologie permettra de comprendre.

\section{b) Aspects critiques et intégristes}

$\underline{\text { Retour à la table des matières }}$

L'analyse détaillée d'un discours idéologique contre-culturel ${ }^{5}$ m'a permis d'identifier un ensemble d'éléments critiques directement ou indirectement reliés à la tendance technocratique. À un premier niveau, cette critique s'articule autour d'une remise en question du phénomène bureaucratique, du recours généralisé aux technologies dures, du gaspillage de l'énergie, d'un certain modèle de développement scientifique et technologique et de la tendance à la centralisation. Plus indirectement, la critique vise des institutions qui assurent le fonctionnement idéologique élémentaire. La famille, les rapports sexuels, l'école sont remis en question dans leur mode de fonctionnement actuel.

Cette critique présente l'avantage de se situer au niveau d'un ensemble de contradictions secondaires, aux points de friction où les luttes sociales ont tendance à se multiplier. Cependant, la critique demeure le plus souvent au niveau des effets manifestes de ces contradictions plutôt qu'au niveau de leur intégration dans un modèle explicatif d'ensemble. L'ensemble de ces critiques est faiblement articulé, sinon à travers une explication totalisante qui déplace les contradictions du niveau des rapports sociaux vers une opposition idéaliste entre l'individu et une société où il est opprimé.

Faute d'un modèle permettant l'analyse dynamique des diverses contradictions, le discours contre-culturel se rabat sur une survalorisation de l'individu-sujet. Les institutions sociales sont représentées comme une force plus ou moins homogène qui est en opposition avec l'individu, lequel devient le centre et la condition même de toute transformation. En effet, il n'est question de transformation sociale que médiatisée par une transformation culturelle, elle-même devant être précédée par une transformation de la conscience individuelle. À la critique des institutions, on oppose la nécessité de renforcer la liberté humaine, de retrouver la richesse de l'individu, de faire renaître les capacités créatrices du sujet. Ce n'est qu'à travers cette révolution des consciences que la critique sociale pourra devenir opérante. À mon point de vue, ce décentrement des contradictions sociales vers le primat de la conscience individuelle tend à renforcer - à l'en-

Jules Duchastel, thèse de doctorat, op. cit. 
contre même du projet critique contre-culturel - l'interpellation du sujet comme catégorie centrale du fonctionnement idéologique.

Un second aspect intégriste de cette idéologie est la contrepartie de ce qui précède. Le discours contre-culturel favorise très explicitement l'apolitisme. À quelques exceptions près ${ }^{6}$, les mouvements sociaux contre-culturels rejettent toute forme d'action politique. De la même manière, le discours contre-culturel s'exclut du terrain des luttes politiques telles qu'elles peuvent se définir traditionnellement ou à travers des modèles révolutionnaires. Cette idéologie est justement caractérisée par cette position qui consiste à remettre en question la pertinence de l'action politique pour les transformations sociales. À la limite, toute forme sociale est oppressive pour l'individu, si celui-ci ne retrouve pas sa propre autonomie.

Si l'on observe dans l'état actuel des formations capitalistes avancées un ensemble de phénomènes qui tendent à renforcer les mécanismes de contrôle : concentration du pouvoir aux niveaux exécutifs, concentration du pouvoir dans des centres géographiques, confusion des pouvoirs exécutifs, législatifs et judiciaires, politisation du personnel d'État, couple réforme/répression, il est paradoxal de proposer un désinvestissement de la lutte sur le terrain de l'affrontement proprement politique - non pas seulement pour le porter aux niveaux des diverses contradictions secondaires - mais pour ramener le tout au niveau de la conscience individuelle.

Il me semble, d'une part, que la contre-tendance idéologique est contre-tendance au point de vue de son lieu d'intervention et de la base sur laquelle elle s'appuie, mais elle s'inscrit, à un point de vue plus général, dans l'ensemble des dispositifs qui tendent à renforcer et l'assujettissement des individus et la concentration du pouvoir dans les formations capitalistes avancées.

6 Par exemple, les Yippies de Gerry Rubin. 


\section{La contre-culture est un mouvement social}

$\underline{\text { Retour à la table des matières }}$

Il n'est pas question pour moi d'entrer, dans le cadre de cette courte intervention, dans le fond du problème de l'analyse des mouvements sociaux au cours des années soixante. Je tiens seulement à faire quelques remarques visant à replacer le phénomène de la contre-culture dans le contexte du développement de ces mouvements. Comme je l'ai dit plus haut, la multiplication des contradictions secondaires, le déplacement du terrain de la lutte des rapports de production vers un ensemble de points de friction à tous les niveaux du vécu, entraîne par le fait même une multiplication des mouvements sociaux. Ceux-ci passent de l'affrontement au niveau des rapports de production et des rapports de force autour de l'enjeu du pouvoir vers des terrains de lutte et des enjeux sectoriels. Un tableau très incomplet donne une idée de ces mouvements selon leur type.

\section{Mouvements sociaux des années soixante} (non exhaustif)

\begin{tabular}{cl}
$\begin{array}{c}\text { Situation } \\
\text { historique }\end{array}$ & \multicolumn{1}{c}{ Larges } \\
& $\begin{array}{l}\text { Mouvement pour les droits civiques } \\
\text { Conjoncturelle }\end{array}$ \\
& $\begin{array}{l}\text { Mouvement anti-guerre } \\
\text { Mouvement étudiant }\end{array}$ \\
& Mouvement contre-culturel \\
Structurelle & Mouvements féministes
\end{tabular}

Objectifs

Restreints

Mouvement de libération des prisonniers

Mouvements urbains

Mouvement de libération homosexuelle

Mouvements idéologiques

Mouvements de sectes religieuses

Ce qui est spécifique du mouvement contre-culturel, c'est sa prétention au cours des années 60 à unifier l'ensemble de ces mouvements. À un certain point de vue, c'est un mouvement qui répond à une contrainte structurelle d'ensemble (développement du capitalisme dans sa phase monopoliste) et qui offre un discours alternatif sur l'ensemble 
des dimensions du social. Il propose, comme je l'ai dit plus haut, un projet d'unification idéologique autour de l'idée d'une transformation globale de la société, mais à travers la révolution préalable des consciences. Enfin, dans une certaine mesure, c'est le mouvement qui a généré le discours idéologique le plus élaboré et le plus complet. L'idéologie de la contre-culture a, dans ce sens, largement dépassé le lieu de sa production. Elle a véhiculé ses éléments dans de larges couches de la petite bourgeoisie, voire même du prolétariat, qui n'ont par ailleurs pas participé de près à quelque mouvement concret que ce soit.

Par contre, cette prétention à fournir un principe unificateur à l'ensemble des mouvements sociaux s'est fondée sur l'opposition entre la conscience individuelle et la société opprimante. L'idéologie de la contre-culture représente en elle-même une stratégie de dispersion. Elle favorise la multiplicité des perspectives, des objectifs et des tactiques, refusant la lutte sur le terrain directement politique. En même temps qu'elle représente l'effet de dispersion réel produit par le régime général de la lutte des classes dans l'état actuel de développement du capitalisme, elle le renforce à défaut de pouvoir permettre l'articulation des luttes et une manière de les recentrer pour permettre une transformation. Cette incapacité de jouer un rôle unificateur se reflète depuis les années 70 dans la décrépitude du mouvement contre-culturel (fuites dans la drogue, vers le sectarisme, la réintégration, etc.). Il est indéniable que les conditions objectives qui prévalent dans les sociétés capitalistes ne sont plus les mêmes qu'à la fin des années soixante. Une crise profonde est larvaire depuis cette époque et entraine des conséquences nouvelles au niveau de l'organisation du pouvoir. Les mouvements sociaux à objectifs larges semblent perdre du terrain par rapport aux mouvements ayant des objectifs plus restreints. L'effet de dispersion est d'autant plus grand. Compte tenu de cette réalité, il n'en demeure pas moins que le mouvement contre-culturel était trop largement fondé sur une perspective idéaliste, visant la dispersion des conflits et se réfugiant dans un resserrement de l'idéologie subjectiviste.

\section{Conclusion}

$\underline{\text { Retour à la table des matières }}$

Les questions soulevées dans la description de cet atelier par rapport à la contreculture et au pouvoir trouvent leur réponse essentielle dans le fait que la contre-culture, en même temps qu'elle n'est pas le fruit d'un hasard historique et que sa forme première est d'abord critique, propose un modèle illusoire (utopique) de transformation sociale. Le primat de la révolution des consciences sur la transformation culturelle et sociale déplace non seulement l'analyse, mais la pratique politique du terrain des luttes concrètes 
vers un espace imaginaire qui, en dernière analyse, sera réinséré dans les rapports sociaux d'exploitation et de domination.

Les critiques de la contre-culture, justes à un premier niveau, ne trouvent pas alors le moyen de leur articulation et l'effet produit est la dispersion. Au contraire, la tendance actuelle à la centralisation du pouvoir se trouve renforcée par l'ignorance volontaire du terrain politique des luttes. Enfin, la révolution des consciences renforce la catégorie première du fonctionnement idéologique dans les formations sociales, celle du sujet.

Jules Duchastel

Département de sociologie

Université du Québec à Montréal

Fin du texte. 